\title{
USING COHERENT SCATTERERS IN TIME SERIES OF HIGH RESOLUTION SAR IMAGES FOR THE MONITORING OF CONSTRUCTION ACTIVITY
}

\author{
C. Villamil Lopez ${ }^{1,2}$, U. Stilla ${ }^{2}$ \\ ${ }^{1}$ German Aerospace Center (DLR), Microwaves and Radar Institute - carlos.villamillopez@dlr.de \\ ${ }^{2}$ Photogrammetry and Remote Sensing, Technical University of Munich (TUM), Germany - stilla@tum.de
}

KEY WORDS: SAR, Time Series, Coherent Scatterers, Urban Areas, Construction

\begin{abstract}
:
In this paper, we present a method for detecting and quantifying changes due to construction activity in time series of high resolution SAR images. We propose to detect these changes by the appearance or disappearance of a large number of coherent scatterers, rather than by looking at changes in SAR amplitude or coherence. These coherent scatterers, which appear mainly in buildings and other man-made structures, will be detected in each individual image by analysing their stability across multiple frequency sub-bands. This implies that long time series are not required, unlike for other similar approaches based in persistent scatterer interferometry, and that this approach can be applied for the monitoring of fast changing areas such as temporary settlements. The presented approach will be applied for monitoring the construction activity taking place during the preparations for two large festivals in Munich, Germany.
\end{abstract}

\section{INTRODUCTION}

High resolution SAR images of urban areas have been widely exploited for a variety of applications. One of such applications is the detection of changes due to construction activity, such as new buildings. This has been typically done by applying SAR change detection approaches that exploit changes in SAR amplitude or a loss of coherence between image pairs. Some of these approaches have been specifically developed for detecting changes in urban areas, such as the ones presented by Yousif and Ban (2014) and Kim et al. (2016). Also, Marin et al. (2015) and Tao and Auer (2016) developed more complex change detection methods that aim to detect exclusively newly constructed or demolished buildings, by including a building backscattering model and SAR simulation with a Digital Surface Model (DSM), respectively.

Besides detecting construction activity by the induced changes in radar backscatter or loss of coherence, these can also be detected by the appearance or disappearance of large numbers of strong point-like scatterers that are characteristic of buildings and other man-made structures. In high-resolution SAR images, these strong point-like scatterers often dominate their respective resolution cells, and their amplitude and phase remain stable over time and also over different frequencies, unlike the more random behaviour exhibited by the distributed scattering mechanisms of natural targets. The temporal stability of these point scatterers is exploited by Persistent Scatterer Interferometry (PSI), introduced by Ferretti et al. (2001). Change detection approaches based on PSI, like the one presented by Yang and Soergel (2018), have been employed to detect newly constructed buildings by the appearance of additional persistent scatterers. However, PSI approaches require very long time series with a large amount of SAR images acquired with the same imaging geometry. Such long time series are often not available, and they are expensive to collect. Additionally, these methods are not well suited for the monitoring of construction activity in fast changing areas, such as temporary settlements like refugee camps, or areas where large events such as festivals take place. To solve these limitations, in this paper we propose to perform the detection of these strong point scatterers (which are often referred to as coherent scatterers) in each individual image of the time series, by exploiting their stability over different frequency sub-bands rather than over time. Once these have been detected for each image, construction activity can be detected and quantified by looking at significant changes in the number of coherent scatterers inside any given area. This approach will be described in section 2, and its feasibility will be demonstrated in section 3 , by applying it to monitor the construction activity before and after two large festivals taking place in Munich, Germany. Results will be shown for a series of repeat-pass acquisitions and also for the comparison of observations acquired from two different orbits. Finally, the conclusions and future work will be outlined in section 4 .

\section{METHOD}

\subsection{Detection of Coherent Scatterers in single SAR images}

Stable point-like scatterers which dominate their resolution-cell are often referred to as coherent scatterers (Sanjuan-Ferrer et al. 2015). Different methods have been presented for the detection of coherent scatterers in a single high-resolution SAR image, such as the ones introduced by Schneider et al. (2006) and Giacovazzo et al. (2008). These methods exploit the high bandwidth of these images by computing multiple sub-looks of reduced bandwidth, and try to detect scatterers that remain stable across these sub-looks. The sub-looks can be computed in range (i.e., different frequency bands) azimuth (i.e., different sub-apertures or Doppler frequencies). Sanjuan-Ferrer et al. (2015) have shown a comparison between four different methods. For its simplicity and good performance, we will apply the phase variance approach introduced by Schneider and Papathanassiou (2009). A brief description of this approach will be provided below.

The phase variance approach exploits sub-looks in range (i.e., with different frequency sub-bands) for the detection of coherent scatterers. Sub-looks can be easily computed for a Single Look Complex (SLC) SAR image as described by the following equation:

$$
I_{\text {sub-look }}=\mathcal{F}^{-1}\left\{\mathcal{F}\{I\} \cdot W_{1} \cdot W_{2}\right\}
$$


where

$$
\begin{aligned}
& \mathcal{F}, \mathcal{F}^{-1}=\text { direct and inverse Fourier transforms } \\
& I, I_{\text {sub-look }}=\text { original and sub-look images } \\
& W_{1}, W_{2}=\text { spectrum weighting functions }
\end{aligned}
$$

The weighting function $W_{1}$ should be the inverse of the window applied during the SAR focusing for sidelobe reduction (which is typically specified in the image metadata), whereas $W_{2}$ will be a rectangular window which will determine the sub-look bandwidth and central frequency.

The idea of the phase variance approach for coherent scatterer detection is to evaluate the phase of each pixel at different frequencies: the phase of an ideal point scatterer will vary linearly with frequency, whereas for clutter it will vary randomly. Therefore, if $N$ sub-look images are computed with uniformly spaced central frequencies and equal bandwidth, the phase difference between consecutive sub-looks should be constant for coherent scatterers. Before computing these phase differences, a one-dimensional phase unwrapping should be performed first along the frequency dimension of the sub-look stack to avoid possible phase jumps of $2 \pi$. For each pixel, a standard deviation of this phase gradient $\left(\sigma_{\varphi}\right)$ can then be computed using the following equation:

$$
\sigma_{\varphi}^{2}=\frac{1}{N-1} \sum_{i=1}^{N-1}\left(\Delta \varphi_{i}-\frac{1}{N-1} \sum_{j=1}^{N-1} \Delta \varphi_{j}\right)^{2}
$$

Where $\Delta \varphi_{i}$ is the phase difference between sub-looks $\mathrm{i}+1$ and $\mathrm{i}$

Finally, thresholding hast to be applied to $\sigma_{\varphi}$, where pixels with a $\sigma_{\varphi}$ below this threshold will be classified as coherent scatterers and the rest as clutter. In addition to this threshold, the other parameters that must be selected are the number of sub-looks and the sub-look bandwidth. Sanjuan-Ferrer et al. (2015) have analysed the effect of this parameters in the method's performance. An example of a SAR image patch and the detected coherent scatterers can be seen below in Figure 1.
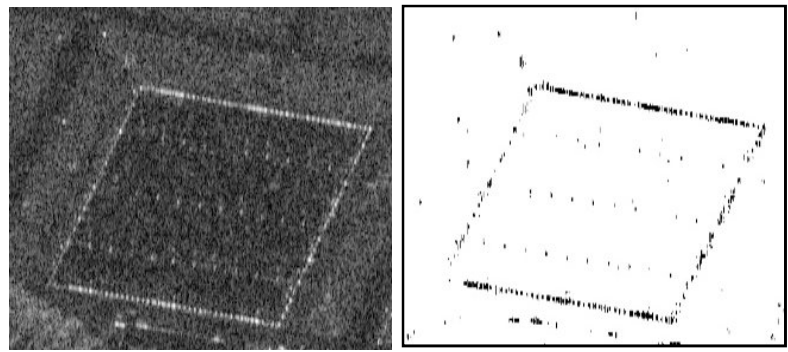

Figure 1. Example results of the coherent scatterer detection: SAR amplitude patch (left) and coherent scatterers (right).

\subsection{Exploiting coherent scatterers for monitoring construction activity in time series}

After the detection of coherent scatterers for each image of the series, a stack of binary images that classify each pixel as either a coherent scatterer or clutter is obtained. Because the detection step must be performed for the original Single Look Complex (SLC) images, co-registration still needs to be performed. This can be done using the geometrical image registration approach introduced by Sansosti et al. (2006). After co-registration, this stack can be analyzed along the temporal dimension. While a few changes are to be expected, the appearance or disappearance of a large number of coherent scatterers in any given area will indicate construction activity, as large numbers of these point scatterers are only present in buildings and other large man-made structures. In contrast, natural targets or small man-made objects such as cars exhibit a much lower number of such scatterers. In section 3, we will show how the evaluation of a simple metric like the number of coherent scatterers inside an area (i.e., its density) gives an accurate image of the construction activity happening inside of it. More elaborate analysis are also possible, such as applying clustering to only take into account groups of closely packed coherent scatterers above a specific size, and ignore isolated points corresponding to some natural targets or smaller man-made objects.

Finally, there is also potential to monitor the height of the detected structures, as it has been shown that the 3D position of coherent scatterers can be determined with high precision. Different methods have been presented for this, such as interferometry without the need of phase unwrapping (Bovenga et al., 2014), or the analysis of the azimuth defocusing in a single image (Duque et al., 2015) or an image pair (Duque et al., 2019).

\section{FIRST RESULTS}

In order to illustrate the presented approach, it will be applied to a series of 12 TerraSAR-X images of Munich, acquired between March and August 2016. All these images were acquired using the Staring Spotlight imaging mode, with a resolution of approx. $25 \mathrm{~cm}$ in azimuth and $60 \mathrm{~cm}$ in slant-range. We will focus on the region of the Theresienwiese Park, where two large festivals take place each year. During the preparations for these events, large tents and other structures are erected, which are later disassembled after the events. The goal is to show that the proposed approach can be exploited to detect and quantify this construction activity.

The acquisition dates of these images are listed in Table 1 together with the corresponding orbits and the events taking

\begin{tabular}{|c|c|c|}
\hline Date & Orbit & Events taking place \\
\hline $\begin{array}{l}28.03 .2016 \\
31.03 .2016\end{array}$ & $\begin{array}{l}\text { Asc. } \\
\text { Desc. }\end{array}$ & None \\
\hline $\begin{array}{l}19.04 .2016 \\
03.05 .2016 \\
\end{array}$ & $\begin{array}{l}\text { Asc. } \\
\text { Desc. }\end{array}$ & $\begin{array}{l}\text { Spring Festival } \\
\text { (Frühlingsfest) }\end{array}$ \\
\hline $\begin{array}{l}22.05 .2016 \\
25.05 .2016 \\
16.06 .2016 \\
24.06 .2016 \\
08.06 .2016 \\
\end{array}$ & $\begin{array}{c}\text { Asc. } \\
\text { Desc. } \\
\text { Desc. } \\
\text { Asc. } \\
\text { Desc. }\end{array}$ & None \\
\hline $\begin{array}{l}16.07 .2016 \\
30.07 .2016 \\
07.08 .2016 \\
\end{array}$ & $\begin{array}{l}\text { Asc. } \\
\text { Desc. } \\
\text { Asc. }\end{array}$ & $\begin{array}{l}\text { Oktoberfest preparations } \\
\text { (starting middle of July) }\end{array}$ \\
\hline
\end{tabular}
place at the Theresienwiese Park at that time. The incidence angles are $37.5^{\circ}$ and $39.4^{\circ}$ for the images acquired during ascending and descending orbits, respectively.

Table 1. Acquisition dates and orbits of the SAR images of Munich, and events in the Theresienwiese during this time.

\subsection{Using repeat-pass acquisitions}

Initially, we will monitor construction activity using only the repeat-pass images acquired with ascending orbit. In order to monitor exclusively the increase/decrease of coherent scatterers inside the Theresienwiese Park, its boundaries were obtained from OpenstreetMap. This polygon was then projected to the SAR image in slant-range geometry by performing geocoding. Coherent scatterers outside of this boundary were ignored. 

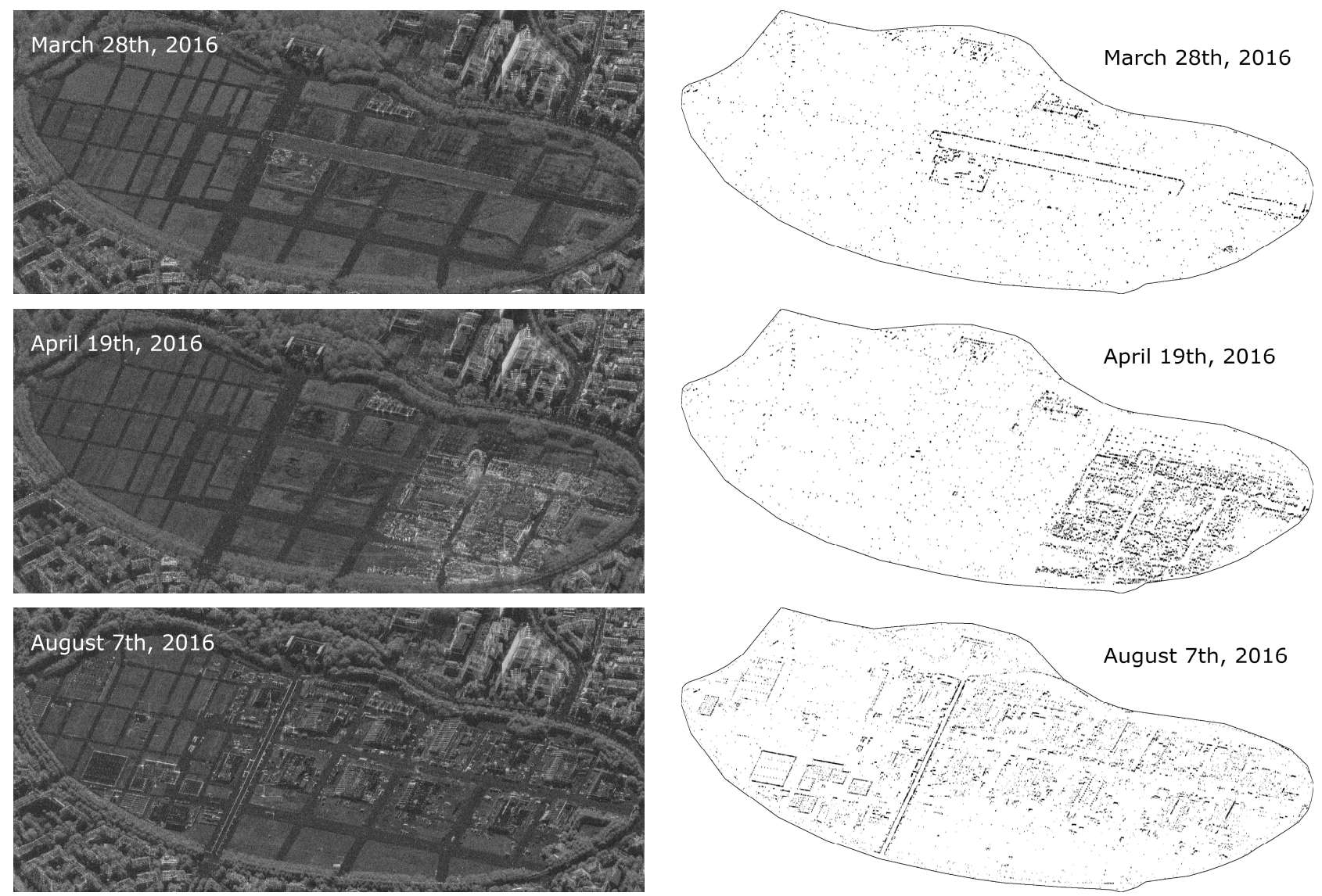

Figure 2. SAR images (left) and coherent scatterers (right) showing the Theresienwiese Park and its state at three different dates: March $28^{\text {th }}$, with no event (top), April $19^{\text {th }}$, during Spring Festival (centre), and August $7^{\text {th }}$, during Oktoberfest preparations (bottom).

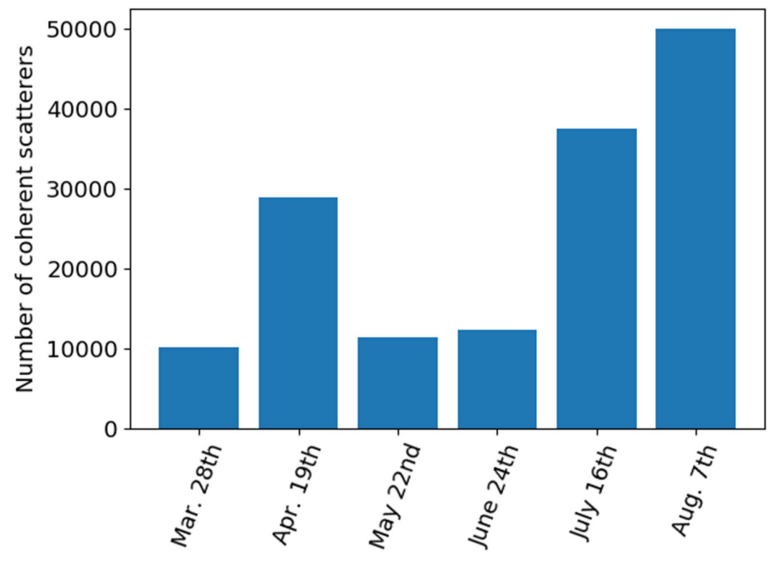

Figure 3. Number of detected coherent scatterers inside the Theresienwiese Park at different dates using repeat-pass images.

Figure 2 shows three SAR images of this park (left) and the corresponding detected coherent scatterers (right) for three different dates. The top, centre and bottom rows correspond to a date when no event is taking place, to a day during the Spring festival (Frühlingsfest), and a day during the construction of the Oktoberfest beer tents, respectively. The number of coherent scatterers detected in the park for each image is shown in the plot of Figure 3. It can be seen that this simple metric correlates well with the construction activity taking place in the park. As expected, a sharp increase in the number of detected coherent scatterers can be observed between March $28^{\text {th }}$ (no event) and April $19^{\text {th }}$ (during the Spring Festival). Additionally, an increasing trend can be seen starting after the middle of July, which is when the preparations for Oktoberfest begin. Also, a higher number of coherent scatterers are detected during the Oktoberfest preparations than during the Spring Festival. This is expected, as the Oktoberfest is a much larger event that extends across the whole park area, rather than just over a part of it. All this can be visually validated by looking at Figure 2 .

\subsection{Comparison of observations from different orbits}

In the previous section we showed how construction activity can be detected and quantified using a time-series of repeat-pass SAR images by looking for significant changes in the amount of coherent scatterers inside a given area. Now, the feasibility of combining observations from different orbits will be explored, as this allows for a more frequent monitoring. Additionally, it enables the possibility to exploit any prior images that might already be available, and which might have been acquired with a different imaging geometry.

Due to the side looking geometry of SAR sensors, using data acquired from different orbits implies that different façades will be seen from each orbit, and the occluded areas will also vary. Because of this the coherent scatterers detected from each orbit will be different. However, given the assumption that buildings typically are somewhat symmetrical, the density of coherent scatterers should remain similar unless there is a change. This assumes the images are acquired by the same sensor and using the same imaging mode, as otherwise the results might not be comparable. 
Figure 4 shows the coherent scatterers detected in two images acquired a week apart from two different orbits. For this comparison, the area under study has been reduced to the overlapping area between both images, as the images acquired in descending orbit did not show the whole park. Even though the detected coherent scatterers are different, they do indeed appear at the same locations and their density remains similar. It is also important to note that some of the differences will be due to changes that occurred during this week, and not only due to the different imaging geometry. Finally, the number of pixels classified as coherent scatterers is shown in the bar plot of Figure 5, this time including the data from both descending orbit (shown in green) and ascending orbit (in blue). Like in the case of repeat-pass images, this simple metric gives an accurate representation of the construction activity occurring inside the park, showing a clear peak during the Spring Festival and an increasing trend during Oktoberfest preparations. Additionally, it can be observed that the measurements from the two different orbits at similar times correlate quite well.
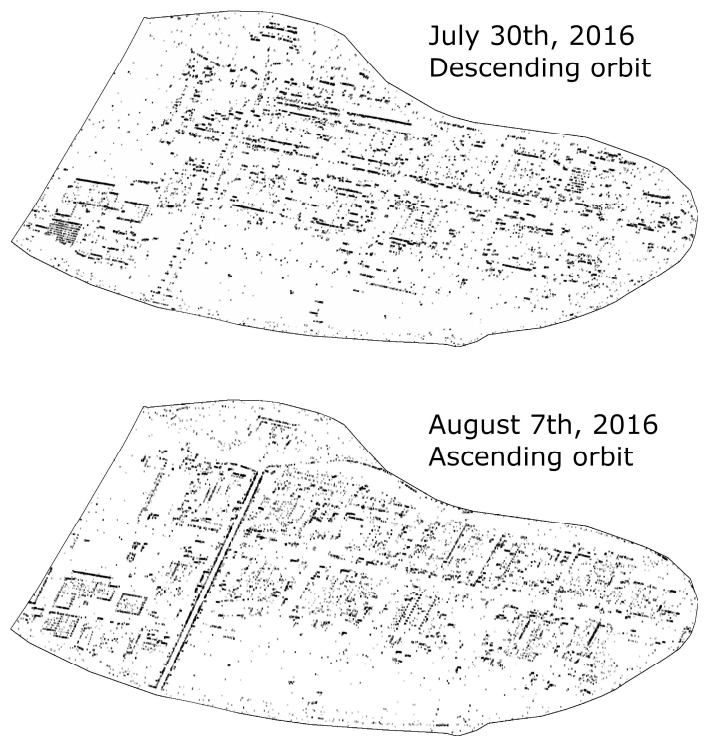

Figure 4. Comparison of the detected coherent scatterers for two images acquired within a week from two different orbits.

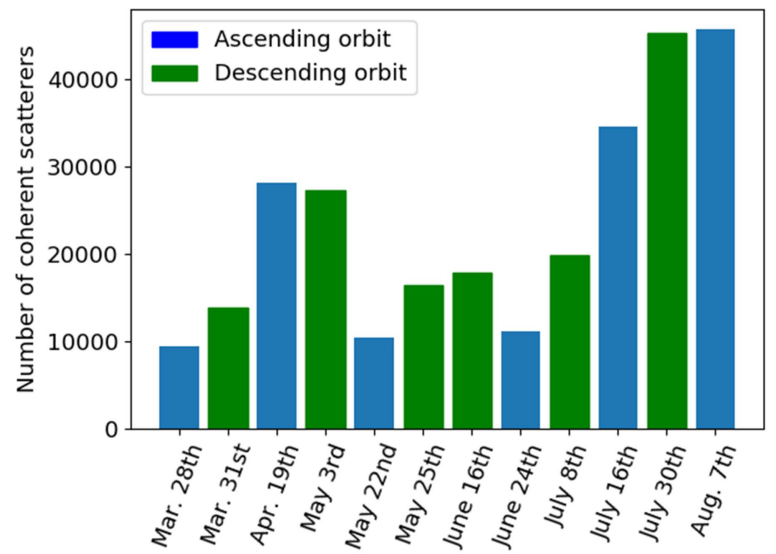

Figure 5. Number of detected coherent scatterers inside the Theresienwiese park at different dates using images acquired from two different orbits.

\section{CONCLUSIONS}

In this paper, we propose to detect and quantify construction activity by looking at the appearance/disappearance of large amounts of strong point scatterers that are present in man-made structures such as buildings. In contrast to persistent scatterer interferometry (PSI) techniques, we detect these coherent scatterers in each individual image by exploiting their stability over the large frequency bandwidth of high resolution images. This gives the important advantage that long time series of repeat-pass high resolution images, which are often not available and are very expensive to collect, are not required for our approach. Additionally, unlike PSI based methods, the proposed method is well suited to monitor fast changing areas, such as temporary settlements (e.g., refugee camps) or locations where large events such as festivals take place. The feasibility of the proposed approach is demonstrated by applying it to detect and quantify the construction activity taking place in the Theresienwiese Park in Munich for the preparation of two large festivals: the Oktoberfest and the Spring Festival. This is shown using a series of repeat-pass images as well as comparison of observations from two different orbits, which allows for more frequent monitoring.

In this paper, we have chosen a rather simple metric for quantifying the construction activity inside a given area: the change in the number of coherent scatterers detected inside it. While it has been shown that this simple metric can give an accurate picture, further work will involve applying more sophisticated methods to quantify the changes due to construction activity from the detected coherent scatterers. One possibility is to apply density based clustering, in a similar way to how it has been described by Villamil Lopez (2018). This can help us to focus on groups of closely packed coherent scatterers above a specific size, while removing isolated coherent scatterers corresponding to smaller objects and/or false detections. Additionally, for the case of repeat pass images, it might also be interesting to first remove the coherent scatterers that remain unchanged, and focus only on the number of coherent scatterers that have either appeared and/or disappeared.

\section{REFERENCES}

Bovenga, F., Rana, F.M., Refice, A., Veneziani, N., 2014. Multichromatic Analysis of Satellite Wideband SAR Data. IEEE Geoscience and Remote Sensing Letters, 11 (10).

Duque, S., Breit, H., Balss, U., Parizzi, A., 2015. Absolute Height Estimation Using a Single TerraSAR-X Staring Spotlight Acquisition. IEEE Geoscience and Remote Sensing Letters, 12 (8), 1735-1739.

Duque, S., Parizzi, A., Zan, F. de, 2019. Precise and Automatic 3-D Absolute Geolocation of Targets Using Only Two LongAperture SAR Acquisitions. IEEE Transactions on Geoscience and Remote Sensing, 1-12.

Ferretti, A., Prati, C., Rocca, F., 2001. Permanent scatterers in SAR interferometry. IEEE Transactions on Geoscience and Remote Sensing, 39(1), 8-20.

Giacovazzo, V. M., Refice, A., Bovenga, F., Veneziani, N., Identification of Coherent Scatterers: Spectral Correlation vs. Multi-Chromatic Phase Analysis, 2018. IGARSS 2008.

Kim, D.-J., Hensley, S., Yun, S.-H., Neumann, M., 2016. Detection of Durable and Permanent Changes in Urban Areas 
Using Multitemporal Polarimetric UAVSAR Data. IEEE Geoscience and Remote Sensing Letters, 13 (2), 267-271.

Marin, C., Bovolo, F., Bruzzone, L., 2015. Building Change Detection in Multitemporal Very High Resolution SAR Images. IEEE Transactions on Geoscience and Remote Sensing, 53 (5), 2664-2682.

Sanjuan-Ferrer, M.J., Hajnsek, I., Papathanassiou, K.P., Moreira, A., 2015. A New Detection Algorithm for Coherent Scatterers in SAR Data. IEEE Transactions on Geoscience and Remote Sensing, 53 (11), 6293-6307.

Sansosti, E., Berardino, P., Manunta, M., Serafino, F., Fornaro, G., 2006. Geometrical SAR image registration. IEEE Transactions on Geoscience and Remote Sensing, 44(10), 2861-2870.

Schneider, R. Z., Papathanassiou, K. P., Hajnsek, I., Moreira, A., 2006. Polarimetric and interferometric characterization of coherent scatterers in urban areas. IEEE Transactions on Geoscience and Remote Sensing, 44(4), 971-984.

Schneider, R. Z., Papathanassiou, K., 2009. Estimation and correction of ionospheric induced phase errors in SAR images using Coherent Scatterers. 2009 IEEE International Geoscience and Remote Sensing Symposium, IV-165-IV-168.

Tao, J., Auer, S., 2016. Simulation-Based Building Change Detection From Multiangle SAR Images and Digital Surface Models. IEEE Journal of Selected Topics in Applied Earth Observations and Remote Sensing, 9(8), 3777-3791.

Villamil Lopez, C., Stilla, U., 2018. Object-based SAR change detection for security and surveillance applications using density based clustering. EUSAR 2018.

Yang, C. H., Soergel, U., 2018. Adaptive 4D Change Detection Based on PSI. EUSAR 2018.

Yousif, O., Ban, Y., 2014. Improving SAR-based urban change detection by combining MAP-MRF classifier and nonlocal means similarity weights. IEEE Journal of Selected Topics in Applied Earth Observations and Remote Sensing, 7(10), 4288-4300. 